\title{
Mesoporous carbon-supported Pd nanoparticles with high specific surface area for cyclohexene hydrogenation: Outstanding catalytic activity of $\mathrm{NaOH}$-treated catalysts
}

\author{
R. Puskás a , T. Varga a , A. Grósz ${ }^{\text {a }}$, A. Sápi a ${ }^{\text {a }}$ A. Oszkó ${ }^{\text {b }}$, Á. Kukovecz ${ }^{\text {a,c }}$, Z. Kónya $^{\text {a,d,* }}$ \\ a Department of Applied and Environmental Chemistry, University of Szeged, Rerrich Béla tér 1, H-6720 Szeged, Hungary \\ b Department of Physical Chemistry and Materials Science, University of Szeged, Aradi Vértanúk tere 1, H-6720 Szeged, Hungary \\ c MTA-SZTE “Lendület" Porous Nanocomposites Research Group, Rerrich Béla tér 1, H-6720 Szeged, Hungary \\ d MTA-SZTE Reaction Kinetics and Surface Chemistry Research Group, Rerrich Béla tér 1, H-6720 Szeged, Hungary
}

\section{A R T I C L E I N F O}

\section{Article history:}

Received 29 May 2015

Received in revised form 15 December 2015

Accepted 16 December 2015

Available online $\mathrm{xxxx}$

\section{Keywords:}

Chemical vapor infiltration

Mesoporous carbon

Palladium nanoparticles

Cyclohexene hydrogenation

\begin{abstract}
A B S T R A C T
Extremely high specific surface area mesoporous carbon-supported Pd nanoparticle catalysts were prepared with both impregnation and polyol-based sol methods. The silica template used for the synthesis of mesoporous carbon was removed by both $\mathrm{NaOH}$ and $\mathrm{HF}$ etching. Pd/mesoporous carbon catalysts synthesized with the impregnation method has as high specific surface area as $2250 \mathrm{~m}^{2} / \mathrm{g}$. In case of $\mathrm{NaOH}$-etched impregnated samples, the turnover frequency of cyclohexene hydrogenation to cyclohexane at $313 \mathrm{~K}$ was obtained $\sim 14$ molecules $\cdot \mathrm{site}^{-1} \cdot \mathrm{s}^{-1}$. The specific surface area of HF-etched samples was higher compared to NaOH-etched samples. However, catalytic activity was 3-6 times higher on $\mathrm{NaOH}$-etched samples compared to HF-etched samples, which can be attributed to the presence of sodium and surface hydroxylgroups of the catalysts etched with $\mathrm{NaOH}$ solution.
\end{abstract}

(c) 2015 Elsevier B.V. All rights reserved.

\section{Introduction}

Amorphous carbon-supported Pd nanoparticles are promising catalysts due to the extremely high specific surface area, which can range from a few hundred to several thousand $\mathrm{m}^{2} / \mathrm{g}$ [1-4]. Also, amorphous carbon structures are easily prepared from almost any organic materials, like nutshells, corn straw, oat hull, sunflower shell, etc., which makes them relatively inexpensive [5].

Catalytic hydrogenation of cyclohexene is a vigorously studied research field of catalysis as the reaction is the basis of several key processes of fuel industry, thus making cheaper, more durable catalysts and acquiring more knowledge about the underlying processes is of paramount importance [6-8].

Several factors in palladium-based catalysts, like the effect of pore structure and metal/support interaction, were studied in recent years [9-11]. The promotion effect of alkali metals on different noble metalbased catalysts has also been investigated [12-16]. It has been found that alkali metals generally have a beneficial effect on both conversion and selectivity on metal oxide-supported catalysts, depending on the

\footnotetext{
* Corresponding author at: Department of Applied and Environmental Chemistry, University of Szeged, Rerrich Béla tér 1, H-6720 Szeged, Hungary.

E-mail address: konya@chem.u-szeged.hu (Z. Kónya).
}

alkali loading concentration. However, the effect of alkali promotion on high surface area activated carbon-supported Pd catalyst prepared with the template method [17] has not been studied earlier to our knowledge.

In this paper, we investigate the catalytic hydrogenation of cyclohexene to cyclohexane in the gas-phase over mesoporous carbonsupported Pd nanoparticles, where the support was prepared with a silica-based template method. The silica template of the high surface area carbon was removed by etching samples in either $\mathrm{NaOH}$ or HF. Pd was loaded by the well-known wet impregnation method and a method based on the premixing of the silica template with as-prepared Pd nanoparticles. Pd/mesoporous carbon samples made by wet impregnation method showed higher activity in cyclohexene hydrogenation compared to the premixing-based catalysts. One of the Pd-loaded mesoporous carbon catalysts based on the impregnation method has reached as high as $2250 \mathrm{~m}^{2} / \mathrm{g}$ specific surface area. In case of $\mathrm{NaOH}$-etched samples based on the impregnation method, the turnover frequency for cyclohexene hydrogenation to cyclohexane at $313 \mathrm{~K}$ has reached $\sim 14$ molecules $\cdot \mathrm{site}^{-1} \cdot \mathrm{s}^{-1}$. Etching with HF has resulted in higher specific surface area then etching with $\mathrm{NaOH}$. However, the turnover frequency was found to be 3-6 times higher on samples etched with $\mathrm{NaOH}$ compared to the HF-treated samples as these samples containing trace amounts of sodium and higher amount of hydroxyl groups as evidenced by $\mathrm{X}$-ray photoelectron spectroscopy. 


\section{Experimental}

\subsection{Materials}

As template material for the carbon structures, Ludox SM30 (30 wt\% silica content) colloid silica sol from Sigma-Aldrich was used. Acetylene (Messer) served as carbon source for the mesoporous carbon supports and was diluted in nitrogen (Messer) during the synthesis. Pd-acetate (Sigma-Aldrich) was the precursor for the catalyst, and all Pd impregnated samples were annealed in Argon (Messer) atmosphere. For the removal of the silica template, either concentrated solution of $\mathrm{NaOH}$ (Molar Chemicals) or HF solution (37 wt\%, Molar Chemicals) was used.

\subsection{Synthesis methods}

A simple schematic is provided in Fig. 1 to help understand all the routes taken to produce samples. The basis of the synthesis method used (chemical vapor infiltration or CVI) for the production of the mesoporous carbon (MC) structures is described elsewhere [17]. Shortly described, an appropriate amount of silica sol was vacuum evaporated to obtain $30 \mathrm{~g}$ of solid silica powder, which was further dried in air at $353 \mathrm{~K}$ overnight. The silica powder was placed in a quartz tube using a quartz boat. The tube was purged with pure nitrogen $(300 \mathrm{ml} / \mathrm{min})$ for $10 \mathrm{~min}$, after which the reactor was placed in the preheated furnace ( $973 \mathrm{~K}$ ) and an acetylene flow (30 $\mathrm{ml} / \mathrm{min}$ ) was introduced on the silica powder for $1 \mathrm{~h}$. After the reaction, purging was performed to continue the carbonization process in pure nitrogen atmosphere for $1 \mathrm{~h}$, where the nitrogen flow was kept at $300 \mathrm{ml} / \mathrm{min}$. Two different temperatures were chosen for the carbonization process ( $973 \mathrm{~K}$ and $1173 \mathrm{~K}$ ) to evaluate the effect of temperature on various properties of the final products. For easy referencing, samples prepared at carbonization temperatures $973 \mathrm{~K}$ and $1173 \mathrm{~K}$ will be denoted as MC77 and MC79, respectively, which stand for mesoporous carbon $700+700{ }^{\circ} \mathrm{C}$ or mesoporous carbon $700+900{ }^{\circ} \mathrm{C}$.

For the removal of the silica templates, two methods were used. One of the method utilized HF solution for the removal of silica, whereas the second method was based on a concentrated solution of $\mathrm{NaOH}$. Shortly described, the as-synthesized carbon-silica composite material ( $30 \mathrm{~g})$ was placed in a plastic vessel and $100 \mathrm{ml}$ of HF solution was dropwisely added. After addition of the HF solution, the mixture was allowed to cool to room temperature and the material was kept in the solution for $24 \mathrm{~h}$. The samples were then washed to neutral $\mathrm{pH}$ with distilled water and dried overnight at $353 \mathrm{~K}$ in air. The procedure was repeated to ensure all silica was removed.

The $\mathrm{NaOH}$ etching was performed in a PTFE-coated steel autoclave (400 $\mathrm{cm}^{3}$ total volume), where the composite material was mixed with $10 \mathrm{~mol} / \mathrm{dm}^{3} \mathrm{NaOH}$ solution. The sealed autoclave was then placed in a furnace preheated to $413 \mathrm{~K}$ for $24 \mathrm{~h}$. Afterwards, the material was cleaned with filtration and was washed with distilled water until neutral pH was reached. The samples were finally dried in air at $353 \mathrm{~K}$ overnight.

Pd nanoparticles were introduced into the mesoporous carbon structures by selecting two different methods. The first method is the wellestablished wet impregnation procedure, where $22 \mathrm{mg}$ of Pd-acetate was dissolved in $100 \mathrm{ml}$ of toluene, and then $200 \mathrm{mg}$ of carbon material was added to the solution. The mixture was ultrasonically treated in an ultrasonic bath for 15 min and then stirred with a magnetic stirrer for $24 \mathrm{~h}$ at room temperature. The solvent was finally separated with centrifugation, and the carbon material was dried overnight at $353 \mathrm{~K}$. Nanoparticles were formed by thermal annealing of the samples in $\mathrm{Ar}$ atmosphere, where the samples were placed in a quartz tube using ceramic crucibles. The tube was purged with pure argon $(300 \mathrm{ml} / \mathrm{min})$ for $10 \mathrm{~min}$ to remove oxygen, after which the tube was placed into a preheated furnace. The furnace was operated at $458 \mathrm{~K}$ for $2 \mathrm{~h}$ then was further heated to $653 \mathrm{~K}$ at a rate of $20 \mathrm{~K} / \mathrm{min}$ and was left there for $1 \mathrm{~h}$. The tube was finally allowed to cool naturally to room temperature outside of the oven. Samples modified with this method have an "-I" suffix in their names (MC77-I and MC79-I).

The second method, which is from now on called the "Pd-sol method," required a separate synthesis of Pd nanoparticles and a slight modification of the original synthesis method of the mesoporous carbon support. Pd nanoparticles were synthesized as described by Nutt et al. [18]. The as-prepared Pd-sol was simply added to the SM-30 colloid silica sol prior to vacuum evaporation, which ensured a very even distribution of nanoparticles. The mixture was then treated as described earlier to obtain the carbon deposition on the sample; no further modifications were made to the procedure. Samples prepared with this method has got a "-C" suffix in their names (MC77-C and MC79-C).

\subsection{Characterization methods}

Powder X-ray diffraction patterns were recorded with a Rigaku Miniflex II desktop diffractometer using $\mathrm{CuK}_{\alpha}$ radiation with a scan speed of $1^{\circ} \cdot \mathrm{min}^{-1}$. Microscopy images were acquired using a FEI Tecnai $\mathrm{G}^{2} 20 \mathrm{X}$-TWIN transmission electron microscope (TEM) with a tungsten cathode operated at $200 \mathrm{kV}$. Nitrogen adsorption isotherms were measured with a Quantachrome NOVA 3000e instrument.

X-ray photoelectron spectra (XPS) were taken with a SPECS instrument equipped with a PHOIBOS 150 MCD 9 hemispherical electron energy analyzer operated in the FAT mode. The excitation source was the $K_{\alpha}$ radiation of an aluminum anode $(h \nu=1486.6 \mathrm{eV})$. The X-ray gun was operated at $210 \mathrm{~W}(14 \mathrm{kV}, 15 \mathrm{~mA})$. The pass energy was set to $20 \mathrm{eV}$, the step size was $25 \mathrm{meV}$, and the collection time in one channel was 250 ms. Typically, 5-10 scans were added to acquire a single spectrum. Energy referencing was not applied, and spectra are represented as acquired. For data acquisition, both manufacturer's (SpecsLab2) and commercial (CasaXPS, Origin) software were used. In all cases, the powder-like samples were evenly laid on one side of a double-sized adhesive tape, the other side being attached to the sample

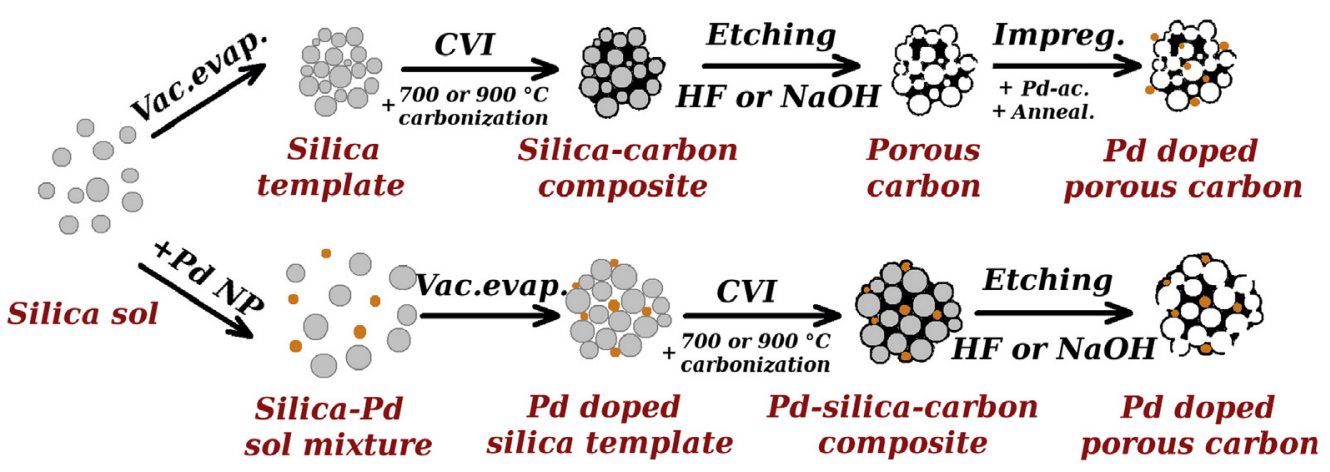

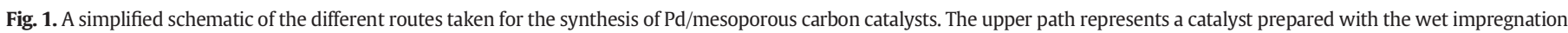
method, while the bottom path shows the "Pd-sol method." 
holder of the XPS instrument. The samples were evacuated at room temperature and then inserted into the analysis chamber of the XPS instrument. No other treatments were applied.

Hydrogen adsorption measurements were performed with a Bel Japan, Inc., BELCAT-A instrument, where samples were activated at $573 \mathrm{~K}$ for $45 \mathrm{~min}$ in hydrogen-nitrogen (1:9) flow (100 ml/min) followed by a $45 \mathrm{~min}$ purging in nitrogen flow $(100 \mathrm{ml} / \mathrm{min})$. Hydrogen adsorption measurements were conducted at $373 \mathrm{~K}$ after activation and purging.

Catalytic hydrogenation of cyclohexene to cyclohexane were performed in a "U"-shaped glass flow reactor at $313 \mathrm{~K}$ with an on-line GC system (Agilent 6820) equipped with FID detection for gas analysis, where the ratio of cyclohexene-hydrogen-nitrogen was 1:10:90 with a total flow rate of $101 \mathrm{ml} \cdot \mathrm{min}^{-1}$ at 1 bar. The only product was cyclohexane during the reactions. The number of active sites for each catalyst was calculated based on the particle size (TEM) and energy dispersive spectroscope (EDS) integrated onto a Hitachi S-4700 scanning electron microscope (SEM), assuming the same atomic density on the Pt nanoparticle surface as Pt (111).

\section{Results and discussion}

\subsection{Nitrogen adsorption}

Nitrogen adsorption measurements were conducted on the raw mesoporous carbon supports and the mesoporous carbon-supported Pd nanoparticle catalysts. Specific surface area $\left(\mathrm{S}_{\mathrm{BET}}\right)$ was calculated with the Brunauer-Emmett-Teller (BET) equation in every case. Mesoporous carbon support carbonized at $900^{\circ} \mathrm{C}$ has a specific surface area of $1746 \mathrm{~m}^{2} / \mathrm{g}$, which value is significantly higher compared to specific surface area values of other carbon allotropes (Fig. 2A). The carbon support carbonized at $700{ }^{\circ} \mathrm{C}$ on the other hand has a specific surface area of $1695 \mathrm{~m}^{2} / \mathrm{g}$, which is not significantly smaller than its $900{ }^{\circ} \mathrm{C}$ counterpart. All the Pd nanoparticle/mesoporous carbon samples have high specific surface area $\left(650-2250 \mathrm{~m}^{2} / \mathrm{g}\right.$ ) as shown on Fig. 2B. Samples synthesized by the impregnation method show higher surface area compared to the samples based on the "Pd-sol method."

A clear difference is visible between HF- and $\mathrm{NaOH}$-etched samples. All HF-etched samples exhibit considerably higher specific surface area compared to $\mathrm{NaOH}$-treated samples. This could be the result of the better efficiency at which HF removes the silica template from the carbon structure, since $\mathrm{SiO}_{2}$ form gaseous $\mathrm{SiF}_{4}$ during the reaction. The reaction between $\mathrm{SiO}_{2}$ and $\mathrm{NaOH}$ is slower and resulting waterglass, a less favorable outgoing component. XPS results show small amount of fluorine in case of HF etching, and showing sodium in samples treated by $\mathrm{NaOH}$. Silicon was not observable in any of the samples.

Higher carbonization temperatures resulted in higher specific surface area, which observation is in agreement with literature data [19].
The method by which the carbon material is modified with Pd also seems to affect $S_{\mathrm{BET}}$ values. If Pd is introduced prior to the carbon synthesis, the specific surface area of the catalysts is lower for the end product compared to samples made by the impregnation method. This might be due to the presence of sodium citrate and tannic acid in the system, which is used for the synthesis of the Pd nanoparticle sol. After vacuum evaporation, the organic content covers the surface of the silica template, resulting in a carbon source that does not decompose in the same manner as acetylene due to the far more complex structure of these substances. This could easily produce a different carbon structure, which would yield lower surface area, if compared to samples synthesized without these organics. This assumption is validated by the carbon structures seen on TEM micrographs as seen on Fig. 3. The presence of Pd during the carbon deposition can have an effect on the decomposition of the acetylene as Pd can act as a catalyst for (de)hydrogenation and cracking.

\subsection{Transmission electron microscopy}

Transmission electron microscopic images were acquired of all Pd modified mesoporous carbon (Fig. 3). The images clearly show the amorphous nature of the carbon structure on all samples; however, some distinct differences are visible between samples modified with different methods. Samples prepared by the impregnation method (MC79-I and MC77-I) show carbon structures that resemble foams, whereas samples prepared with the "Pd-sol method" (MC79-C and $\mathrm{MC77-C)}$ clearly have a more dense structure. This is due to the leftover organics and/or the catalytic effect of Pd as described earlier. The etching agents on the other hand do not seem to have any fundamental effect on the general look of the materials, and only the specific surface area values are different.

Pd nanoparticles are well dispersed within the carbon structure, and no separate particles are visible. Average particle diameters are plotted on Fig. 4. The diameter of particles supported on MC79-C and MC77-C samples are 8-8.9 $\mathrm{nm}$ and 4.2-5.6 $\mathrm{nm}$, respectively, which are about twice as large as particles supported on their impregnated counterparts MC79-I and MC77-I with diameters 3.4-3.6 nm and 3-4.2 nm, respectively. Since the original average diameter of Pd nanoparticles in the Pd-sol was $4.9 \mathrm{~nm}$, it can be assumed that the increased size is the result of the sintering of the nanoparticles at high temperatures of the mesoporous carbon synthesis. It is also noteworthy that the size of nanoparticles on all HF-etched samples prepared with the "Pd-sol method" is slightly smaller than on the $\mathrm{NaOH}$-etched ones, which may be a result of the slow dissolution of the Pd by the concentrated HF solution.

Such a clear difference is not evident on samples modified with impregnation. Particles on samples MC79-I are virtually identical regardless of the type of the etching method. However, catalysts prepared at lower carbonization temperature (MC77-I) show a larger diameter
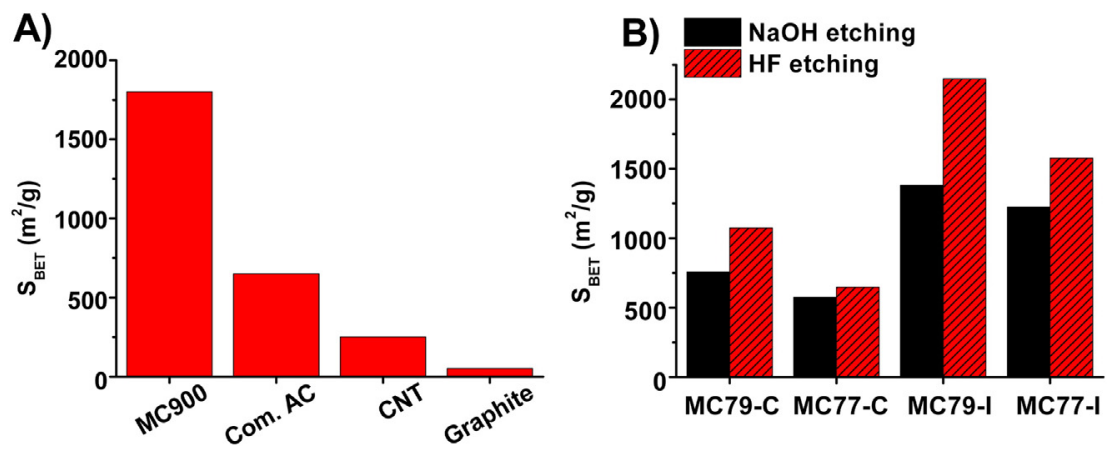

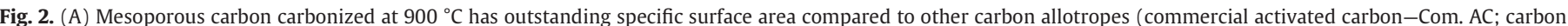

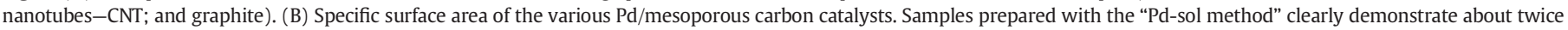

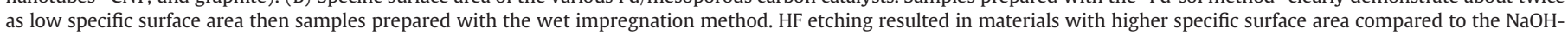
treated catalysts. 

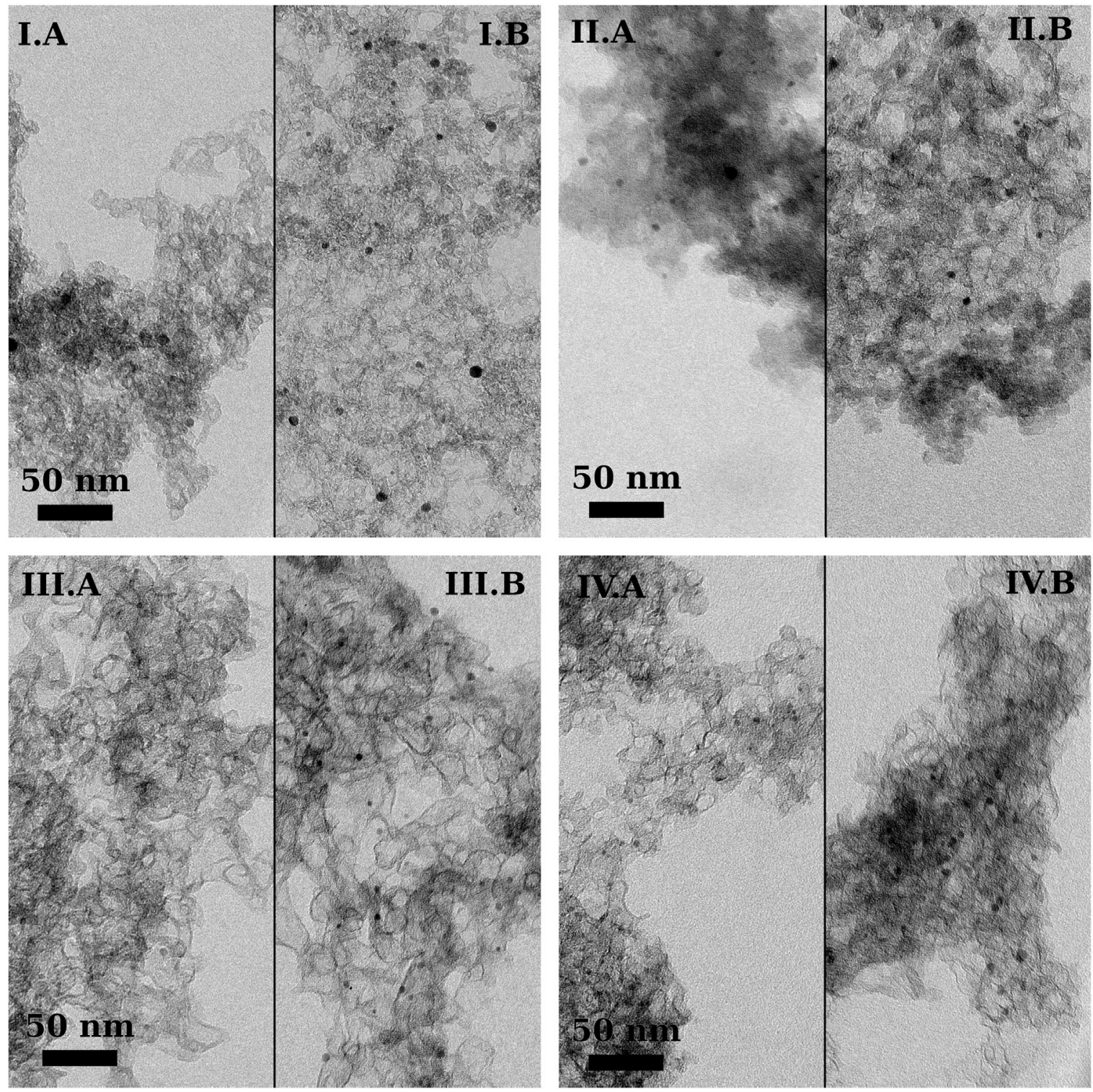

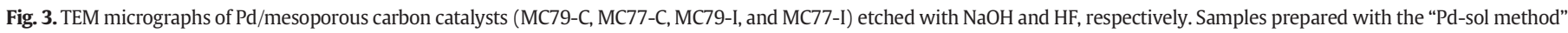

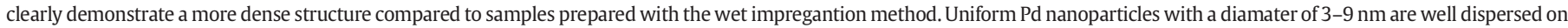
the various mesoporous carbon supports in case of all catalysts.

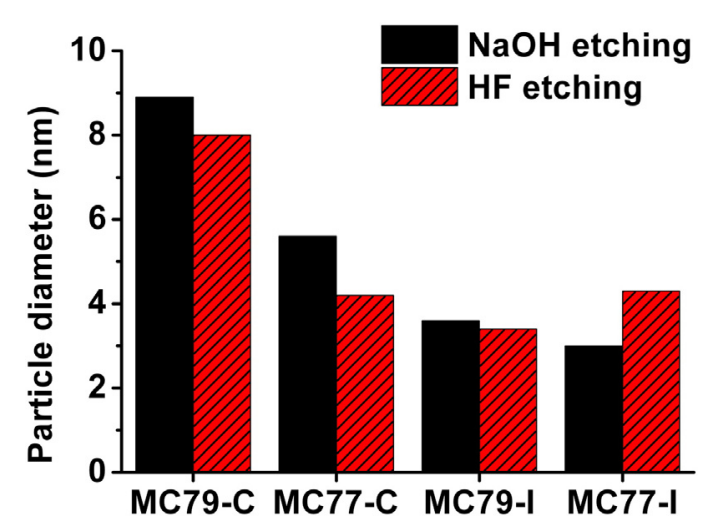

Fig. 4. Average diameter of Pd nanoparticles supported on various mesoporous carbon supports. Samples made by impregnation resulted in 3.4-4.2 nm Pd nanoparticles; however, premixing of the as-synthesized Pd nanoparticles with the silica template (Pdsol method) resulted in larger particle diameter $(4.2-8.9 \mathrm{~nm})$ due to the sintering of the particles at higher carbonization temperature. Etching method has no significant effect on the particle diameter. for the HF-etched carbon structure. This difference could mean that higher carbonization temperatures indeed give more stable carbon structures for the Pd nanoparticles.

\subsection{X-ray photoelectron spectroscopy}

All four mesoporous carbon-supported Pd nanoparticle catalysts (MC79-C, MC77-C, MC79-I, and MC77-I) were investigated by XPS. Low resolution survey, and high resolution $\mathrm{O} 1 \mathrm{~s}, \mathrm{C} 1 \mathrm{~s}$, Na $1 \mathrm{~s}$, and $\mathrm{F} 1 \mathrm{~s}$ (where relevant) spectra were taken. Since the spectra obtained were practically energy correct, no further calibration was applied. The $01 \mathrm{~s}$ signal can be fitted with three components in all cases. The most intense component is at $534.3 \mathrm{eV}$, supposedly from water, but in $\mathrm{NaOH}$-treated samples, the most intense signal is at $532.5 \mathrm{eV}$, due to $\mathrm{OH}$ groups.

Very intense strongly asymmetric $C 1 \mathrm{~s}$ spectra were recorded in all four Pd-loaded samples. The peak maxima were at $285.1 \mathrm{eV}$, characteristic of amorphous carbon. At least three or even more components could be identified on the high binding energy side of the spectra. These all originate from various carbon-oxygen groups, among them from $-\mathrm{COOH}$. 

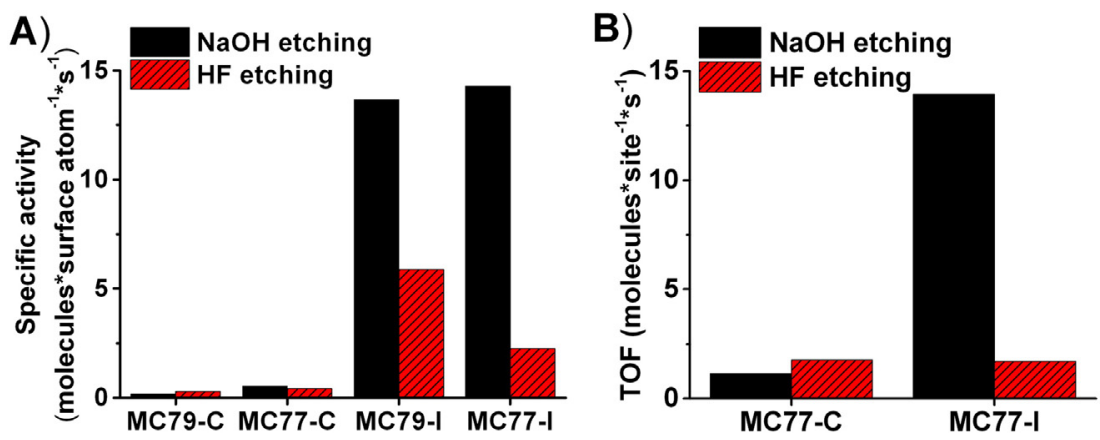

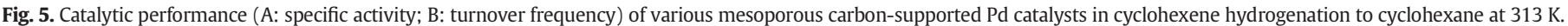

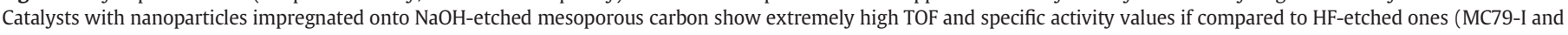

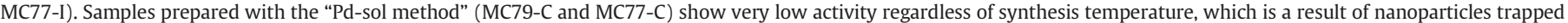
into the pores of the support during the carbonization process of the mesoporous carbon synthesis.

Weak signal maxima between 1072.3 and $1072.6 \mathrm{eV}$ can be attributed to sodium in $\mathrm{NaOH}$-etched samples. The high binding energy reveals that sodium is very positive, possibly in the form of oxide, hydroxide, or halogenide. Fluorine 1s spectra were also taken in relevant samples. This very low intensity signal peaks at $689.7 \mathrm{eV}$, marking a very positive fluorine ion. This binding energy is much higher than found in alkali fluorides but matches the values found in C-F-containing compounds.

\subsection{Catalytic tests}

Catalytic hydrogenation of cyclohexene to cyclohexane was performed on all Pd-loaded mesoporous carbon. Both specific activity and turnover frequencies (TOF) were calculated (Fig. 5), where for specific activity calculation, all surface atoms were assumed to be active centers in the conversion process. For TOF value calculations, the number of active centers was determined by hydrogen chemisorption technique. Calculation methods, data, and tabulated results for both specific activity and TOF are provided in the supplementary information.

Results indicate that samples prepared with the "Pd-sol method" (MC79-C and MC77-C) has low catalytic activity, as specific activity values of these samples do not exceed a value of 0.5 molecules. surface atom $^{-1} \cdot \mathrm{s}^{-1}$. Samples prepared with the wet impregnation method, however (MC79-I and MC77-I), show high specific activity values of 2.2-5.9 and 13.7-14.3 molecules - surface atom ${ }^{-1} \cdot \mathrm{s}^{-1}$. The former related to samples etched by HF and the latter values correspond to catalysts prepared with $\mathrm{NaOH}$ etching. To support these results, TOF values were also calculated for MC77 samples. Results indicate that samples prepared with the "Pd-sol method" (MC77-C) showed relatively low values of 1.1 and 1.7 molecules $\cdot$ site $^{-1} \cdot \mathrm{s}^{-1}$ for both $\mathrm{NaOH}$ - and HF-treated samples, respectively. The $\mathrm{NaOH}$-treated sample (MC77-I) prepared with the wet impregnation method, however, showed a TOF value of 13.9 molecules $\cdot \operatorname{site}^{-1} \cdot \mathrm{s}^{-1}$, whereas the HF-treated sample has a value of 1.7 molecules $\cdot \operatorname{site}^{-1} \cdot \mathrm{s}^{-1}$. The low specific activity values and also the low hydrogen adsorption-based dispersion of samples prepared by the "Pd-sol method" could be the result of several factors. The most obvious factor is that the Pd nanoparticles covered by the mesoporous carbon getting trapped into the pores as they premixed with the silica template prior to the carbon deposition. The presence of organic stabilizing agent originated from the Pd nanoparticle synthesis can result in active site blocking. This assumption is supported by both BET and TEM studies as described earlier. The other possible reason for low specific activity values could be the enlarged nanoparticle diameters due to sintering of the particles at the high carbonization temperatures of the mesoporous carbon synthesis. Interestingly, calculated TOF values do not seem to show a notable difference between samples prepared with different Pd modification techniques, which means that on available active sites, the reaction mechanism is identical for these samples despite the presence of surface coverage.
Impregnated samples etched by $\mathrm{NaOH}$ exhibit outstandingly high specific activity and TOF values ( 14$)$, which is several times higher than normal TOF values found in other reports (Table 1$)$. This agreement in TOF and specific activity supports the assumption of a possible Na promotion, as the TOF values for all the other samples do not seem to deviate from the values 1.1-1.7 molecules $\cdot \mathrm{site}^{-1} \cdot \mathrm{s}^{-1}$. These extremely high values however are only found on samples etched with $\mathrm{NaOH}$, despite the fact that the $\mathrm{S}_{\mathrm{BET}}$ values of these materials are $25-35 \%$ lower than the values of impregnated samples etched with HF. This extraordinary leap in TOF is possibly a promoting effect of sodium which presents in the carbon structure as evidenced by XPS data. An elevated concentration of hydroxyl groups was also found in $\mathrm{NaOH}$-etched samples, which could also contribute to these high TOF values. Interestingly, the fluorine-containing groups found in HF-etched samples did not seem to have any major effect on the catalytic activity of these catalysts.

\subsection{Conclusion}

Mesoporous carbon-supported Pd nanoparticles prepared with both impregnation and "Pd-sol method" were tested in cyclohexene hydrogenation to cyclohexane at $313 \mathrm{~K}$. Samples prepared with the "Pd-sol method" showed very low specific activity values regardless of mesoporous carbon synthesis temperature, which can be attributed to the amorphous carbon coverage of the Pd nanoparticles formed during high temperature carbon synthesis. Specific surface area values were $\sim 2$ times lower for such samples if compared to samples prepared with the wet impregnation method. $\mathrm{HF}$ and $\mathrm{NaOH}$-etched samples prepared with the wet impregnation method on the other hand showed specific activity values of $\sim 2-5$ molecules $\cdot$ surface atom ${ }^{-1} \cdot \mathrm{s}^{-1}$ and $\sim 14$ molecules surface atom ${ }^{-1} \cdot \mathrm{s}^{-1}$, respectively. Samples etched by $\mathrm{HF}$ resulted in higher specific surface area compared to $\mathrm{NaOH}$-etched samples. However, specific activity values were 3-6 times higher on samples etched by $\mathrm{NaOH}$ compared to HF-etched samples, which clearly demonstrates the promoting effect of $\mathrm{Na}$ and high concentration of hydroxyl groups evidenced by XPS. This assumption is also validated by calculated TOF values, as the $\mathrm{NaOH}$-treated sample prepared with wet impregnation method also showed an outstandingly high value. Carbonization temperature has a slight effect on the mesoporous carbon support as well as on the specific surface area values of them. In case

Table 1

Literature data for cyclohexene conversion on different Pd catalysts.

\begin{tabular}{ll}
\hline Catalyst & $\mathrm{TOF}\left(\mathrm{s}^{-1}\right)$ \\
\hline $\mathrm{Pd} /$ carbon [20] & 1.4 \\
$\mathrm{Pd} /$ carbon [21] & 0.8 \\
$\mathrm{Pd} / \mathrm{SiO}_{2}[22]$ & 3.2 \\
$\mathrm{Pd} / \mathrm{Al}_{2} \mathrm{O}_{3}[23]$ & 5.3 \\
\hline
\end{tabular}


of HF-etched samples made by the impregnation method, higher carbonization temperature $\left(900{ }^{\circ} \mathrm{C}\right)$ resulted in twice as high TOF compared to samples carbonized at $700{ }^{\circ} \mathrm{C}$, which could be a result of the higher specific surface area of the carbon structure synthesized at $900{ }^{\circ} \mathrm{C}$. Future works will be oriented on the true nature of the outstanding effect of sodium and hydroxyl groups.

\section{Acknowledgment}

This research was supported by the European Union and the State of Hungary, co-financed by the European Social Fund in the framework of TÁMOP-4.2.4.A/ 2-11/1-2012-0001 "National Excellence Program."

This paper was also supported by the János Bolyai Research Scholarship of the Hungarian Academy of Sciences of SA.

\section{Appendix A. Supplementary data}

Supplementary data to this article can be found online at http://dx. doi.org/10.1016/j.susc.2015.12.020.

\section{References}

[1] A.R. Reed, P.T. Williams, Thermal processing of biomass natural fibre wastes by pyrolysis, Int. J. Energy Res. 28 (2004) 131.

[2] W.M.A.W. Daud, W.S.W. Ali, Comparison on pore development of activated carbon produced from palm shell and coconut shell, Bioresour. Technol. 93 (2004) 63.

[3] K. Yang, J. Peng, C. Srinivasakannan, L. Zhang, H. Xia, X. Duan, Preparation of high surface area activated carbon from coconut shells using microwave heating, Bioresour. Technol. 101 (2010) 6163.

[4] Z. Geng, C. Zhang, D. Wang, X. Zhou, M. Cai, Pore size effects of nanoporous carbons with ultra-high surface area on high-pressure hydrogen storage, J. Energ. Chem. 24 (2015) 1 .

[5] O. Ioannidou, A. Zabaniotou, Agricultural residues as precursors for activated carbon production-a review, Renew. Sust. Energ. Rev. 11 (2007) 1966.

[6] E.E. Gonzo, M. Boudart, Catalytic hydrogenation of cyclohexene: 3. Gas-phase and liquid-phase reaction on supported palladium, J. Catal. 52 (1978) 462.

[7] G.A. Somorjai, J.Y. Park, Colloid science of metal nanoparticle catalysts in 2D and 3D structures. Challenges of nucleation, growth, composition, particle shape, size control and their influence on activity and selectivity, Top. Catal. 49 (2008) 126.
[8] R.M. Rioux, B.B. Hsu, M.E. Grass, H. Song, G.A. Somorjai, Influence of particle size on reaction selectivity in cyclohexene hydrogenation and dehydrogenation over silicasupported monodisperse Pt particles, Catal. Lett. 126 (2008) 10.

[9] E. Esmaeili, A.M. Rashidi, Y. Mortazavi, A.A. Khodadadi, M. Rashidzadeh, SMFssupported Pd nanocatalysts in selective acetylene hydrogenation: pore structuredependent deactivation mechanism, J. Energ. Chem. 22 (2013) 717.

[10] M. Martin-Martinez, A. Álvarez-Montero, L.M. Gómez-Saineroa, R.T. Baker, J. Palomar, S. Omara, S. Eser, J.J. Rodriguez, Deactivation behavior of Pd/C and Pt/C catalysts in the gas-phase hydrodechlorination of chloromethanes: structure-reactivity relationship. Appl. Catal. B 162 (2015) 532.

[11] E. Diaz, A.F. Mohedano, J.A. Casas, L. Calvo, M.A. Gilarranz, J.J. Rodriguez, Deactivation of a Pd/AC catalyst in the hydrodechlorination of chlorinated herbicides, Catal. Today 241 (2015) 86.

[12] M. Konsolakis, N. Macleod, J. Isaac, I.V. Yentekakis, R.M. Lambert, Strong promotion by $\mathrm{Na}$ of $\mathrm{Pt} / \gamma-\mathrm{Al}_{2} \mathrm{O}_{3}$ catalysts operated under simulated exhaust conditions, J. Catal. 193 (2000) 330.

[13] N. Mahata, K.V. Raghavan, V. Vishwanathan, Infuence of alkali promotion on phenol hydrogenation activity of palladium/alumina catalysts, Appl. Catal. A 182 (1999) 183.

[14] S. Tracey, A. Palermo, J.P.H. Vazquez, R.M. Lambert, In situ electrochemical promotion by sodium of the selective hydrogenation of acetylene over platinum, J. Catal. 179 (1998) 231.

[15] K. Lin, A. Ko, Na promotion of Pd/MgO catalysts for low-pressure one-step synthesis of MIBK from acetone $+\mathrm{H}_{2}$, Appl. Catal. A 147 (1996) L259.

[16] Y.H. Park, G.L. Price, Promotional effects of potassium on Pd $/ \mathrm{A}_{2} \mathrm{O}_{3}$ selective hydrogenation catalysts, Ind. Eng. Chem. Res. 31 (1992) 469.

[17] E. Horvath, R. Puskas, R. Remias, M. Mohl, A. Kukovecz, Z. Konya, I. Kiricsi, A nove catalyst type containing noble metal nanoparticles supported on mesoporous carbon: synthesis, characterization and catalytic properties, Top. Catal. 52 (2009) 1242

[18] M.O. Nutt, K.N. Heck, P. Alvarez, M.S. Wong, Improved Pd-on-Au bimetallic nanoparticle catalysts for aqueous-phase trichloroethene hydrodechlorination, Appl. Catal. B 69 (2006) 115

[19] W. Li, K. Yang, J. Peng, L. Zhang, S. Guo, H. Xia, Effects of carbonization temperatures on characteristics of porosity in coconut shell chars and activated carbons derived from carbonized coconut shell chars, Ind. Crop. Prod. 28 (2008) 190.

[20] S.D. Jackson, G.J. Kelly, S.R. Watson, R. Gulickx, Cycloalkene hydrogenation over palladium catalysts, Appl. Catal. A 187 (1999) 161.

[21] C.-B. Hwang, Y.-S. Fu, Y.-L. Lu, S.-W. Jang, P.-T. Chou, C.R.C. Wang, S.J. Yu, Synthesis, characterization, and highly efficient catalytic reactivity of suspended palladium nanoparticles, J. Catal. 195 (2000) 336

[22] L.M. Rossi, F.P. Silva, L.L.R. Vono, P.K. Kiyohara, E.L. Duarte, R. Itri, R. Landersc, G. Machado, Superparamagnetic nanoparticle-supported palladium: a highly stable magnetically recoverable and reusable catalyst for hydrogenation reactions, Green Chem. 9 (2007) 379.

[23] E.E. Gonzo, M. Boudart, Catalytic hydrogenation of cyclohexene 3. Gas-phase and liquid-phase reaction on supported palladium, J. Catal. 52 (1978) 462 